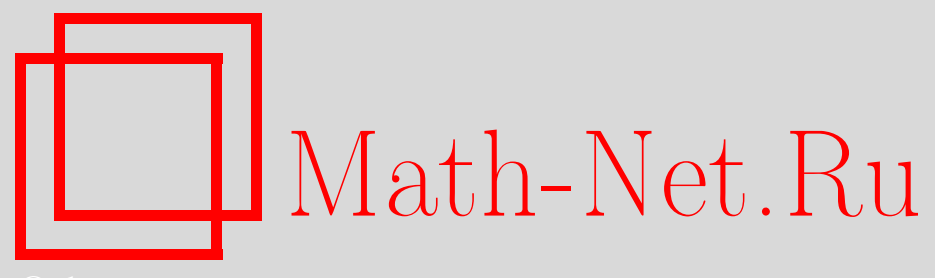

Н. Н. Кузюрин, А. И. Поспелов, Вероятностный анализ шельфовых алгоритмов упаковки прямоугольников в полосу, Дискрет. матем., 2006, том 18, выпуск 1, 76-90

DOI: https://doi.org/10.4213/dm33

Использование Общероссийского математического портала Math-Net.Ru подразумевает, что вы прочитали и согласны с пользовательским соглашением http: //www.mathnet.ru/rus/agreement

Параметры загрузки:

IP : 54.198 .64 .247

26 апреля 2023 г., 07:10:59

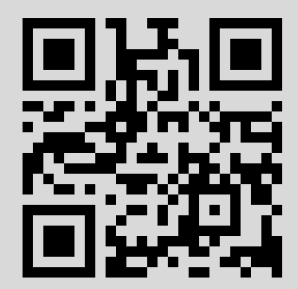




\title{
Вероятностный анализ шельфовых алгоритмов упаковки прямоугольников в полосу
}

\author{
( 2006 г. Н. Н. Кузюрин, А. И. Поспелов
}

\begin{abstract}
Работа посвящена анализу точности приближенных алгоритмов упаковки прямоугольников в полосу. Главным результатом является построение алгоритма, который размещает прямоугольники по мере поступления и для которого отношение математического ожидания незаполненной площади к математическому ожиданию заполненной площади стремится к нулю с ростом числа прямоугольников.

Работа выполнена при поддержке Российского фонда фундаментальных исследований, проекты 05-01-00798 и 04-01-00359.
\end{abstract}

\section{1. Введение}

Задача упаковки прямоугольников в полубесконечную полосу заключается в следующем. Для множества прямоугольников, каждый из которых задан своей высотой и шириной, требуется найти ортогональное размещение прямоугольников (без пересечений и без вращений) в этой полосе, минимизирующее полную высоту этого размещения, то есть максимум по всем прямоугольникам от дна полосы до верхней грани прямоугольника.

Таким образом, входом задачи является полоса $C$ ширины $W$ и список прямоугольников $L=\left\{p_{1}, \ldots, p_{n}\right\}$, причем прямоугольник $p_{i}$ имеет высоту $h_{j}$ и ширину $w_{j}$.

Эта задача возникает в различных ситуациях, и некоторые ее приложения связаны с составлением расписаний для многопроцессорных систем (кластеров). Прямоугольник при этом соответствует описанию вычислительной задачи, ширина определяет необходимые ресурсы (число необходимых процессоров), а высота - время выполнения задачи. Ширина полосы соответствует общему числу процессоров на вычислительном устройстве.

Многие классические задачи теории расписаний являются частными случаями данной задачи. Так, если ширина полосы произвольна (в частности, равна 1), а высоты всех прямоугольников из входного списка равны, то получаем задачу об упаковке в контейнеры, где высота заполнения как раз и равна числу контейнеров [6]. Эти задачи интенсивно исследовались ранее. В общей постановке задача об упаковке в полосу также привлекала внимание исследователей с начала 80-х годов [2].

Известно, что эта задача NP-трудна. Интерес поэтому представляет построение приближенных алгоритмов с гарантированными оценками точности. Различные приближенные алгоритмы были предложены для упаковки прямоугольников в полосу, начиная с 3-приближенного алгоритма [2]. Затем этот результат последовательно улучшался в ряде работ $[1,5,12]$ и в $[10]$ был предложен $(1+\varepsilon)$-приближенный полиномиальный алгоритм 
(для произвольного фиксированного $\varepsilon>0$ ). Последний алгоритм весьма сложен и основан на анализе соответствующих целочисленных линейных программ и на специальных методах округления. К тому же, эти алгоритмы требуют предварительной обработки всего списка прямоугольников, то есть не являются on-line алгоритмами. С точки же зрения приложений интерес представляют алгоритмы, которые размещают очередной прямоугольник из списка без знания последующих прямоугольников, то есть on-line алгоритмы.

Кроме того, в последние годы возрастает интерес к анализу алгоритмов не по худшему случаю, а в среднем, при заданном вероятностном распределении входных данных. Такие исследования интенсивно проводятся в последние годы для одномерной задачи упаковки в контейнеры $[8,7]$.

В данной работе исследуются некоторые простые эвристики для упаковки прямоугольников в полосу, в которых прямоугольники размещаются по мере поступления (то есть в режиме on-line). Наш анализ качества алгоритмов в среднем заключается в оценке математического ожидания незаполненной площади после работы конкретного алгоритма (от основания полосы до верхней границы прямоугольников). Очевидно, что в случае, когда эта площадь равна нулю, упаковку можно считать совершенной, и чем меньше отношение незаполненной площади к заполненной, тем выше качество упаковки. Таким образом, интерес представляет сравнение незаполненной и заполненной площадей.

Главным результатом работы является построение алгоритма, который размещает прямоугольники по мере поступления и для которого отношение математического ожидания незаполненной площади к математическому ожиданию заполненной площади стремится к нулю с ростом числа задач.

\section{2. Описание алгоритма и полученные результаты}

В данной статъе рассматриваются двухэтапные (шельфовые) алгоритмы упаковки [3]. Упаковка состоит из серии слоев (шельфов). Высоты слоев выбираются из некоторого заданного конечного или бесконечного множества $\left\{r_{n}\right\}$ такого, что $r_{n+1}<r_{n}, r=1,2, \ldots$ Прямоугольники упаковываются на основания подходящих слоев, минимальных по высоте, в которые они входят.

Этап 1. Прямоугольники упаковываются в минимальные по высоте шельфы, в которые они входят, то есть, если высота прямоугольника $h_{i}$, упаковываем его в шельф высоты $r_{n}$ такой, что

$$
r_{n+1}<h_{i} \leqslant r_{n}
$$

Этап 2. Упаковка в слои заданной ширины и порождение новых шельфов осуществляется следующей эвристикой, называемой Best-Fit (BF) (см. [7]). Для упаковки выбирается подходящий шельф с минимальной по ширине пустой площадью, в котором можно разместить данный прямоугольник, то есть, если суммарная ширина прямоугольников, размещенных в $i$-м шельфе, равна $t_{i}$, то при размещении $j$-го прямоугольника ширины $w_{j}$ выбирается подходящий по высоте шельф $i_{0}$ такой, что

$$
i_{0} \in \operatorname{Argmin}_{i}\left\{W-t_{i} \mid w_{j} \leqslant W-t_{i}\right\} .
$$

Если для поступившего прямоугольника $j$ нет ни одного подходящего шельфа или среди подходящих шельфов не нашлось такого шельфа $i$, что $w_{j} \leqslant W-t_{i}$, то создается новый шельф с основанием на верхней границе последнего из созданных шельфов и с минимальной высотой $r_{n} \geqslant h_{j}$, в который помещается прямоугольник $j$. 
Мы будем называть алгоритмом A1 шельфовый алгоритм упаковки, который на втором этапе использует эвристику Best-Fit. В дальнейшем будем полагать

$$
r_{n}=(1-\delta)^{n}, \quad 0<\delta<1,
$$

где $\delta$ - некоторый параметр.

Мы будем рассматривать следующие вероятностные распределения: равномерное распределение $U([0, W])$ на отрезке $[0, W]$ и равномерное дискретное распределение $U(\{1,2, \ldots, W\})$ на множестве $\{1,2, \ldots, W\}$.

Всюду в дальнейшем будем считать, что для каждого прямоугольника высота $h_{i}$ имеет равномерное распределение на отрезке $[0,1]$, а для ширины мы рассмотрим два случая, когда ширина каждого прямоугольника имеет равномерное распределение на отрезке $[0, W]$ и когда ширина каждого прямоугольника имеет равномерное распределение на множестве $\{1,2, \ldots, W\}$. Очевидно, что в случае равномерного непрерывного распределения ширины ее можно нормировать, сделав равной 1. Будем предполагать, что все случайные величины $w_{i}, h_{i}$ независимы.

Мы будем оценивать математическое ожидание площади, не заполненной прямоугольниками, между основанием полосы и верхней границей самого верхнего шельфа. В дальнейшем будем обозначать эту величину $\Sigma$. Будем предполагать, что число прямоугольников $N \rightarrow \infty$.

Теорема 1. Для алгоритма Al при распределении ширины прямоугольников по закону $U([0,1])$ справедлива оченка

$$
\Sigma=O\left(\frac{\sqrt{N} \log ^{3 / 4} N}{\sqrt{\delta}}+\delta N\right) .
$$

Если

$$
\delta^{-1}=o(\sqrt{N}), \quad \delta \rightarrow 0,
$$

mo

$$
\Sigma=\Omega\left(\frac{\sqrt{N} \log ^{3 / 4} N}{\sqrt{\delta}}+\delta N\right)
$$

Выбирая

$$
\delta=N^{-1 / 3} \log ^{1 / 2} N
$$

получаем оченку

$$
\Sigma=\theta\left(N^{2 / 3} \log ^{1 / 2} N\right) .
$$

Теорема 2. Для алгоритма А1 при распределении ширины прямоугольников по закону $U(\{1,2, \ldots, W\})$ справедлива оченка

$$
\Sigma=O\left(\frac{\sqrt{N} \log ^{3 / 4} W}{\sqrt{\delta}}+\delta W N\right)
$$

Если

$$
\delta^{-1}=o(N), \quad \delta \rightarrow 0
$$


mo

$$
\Sigma=\Omega\left(\frac{\sqrt{N} \log ^{3 / 4} W}{\sqrt{\delta}}+\delta W N\right)
$$

Bыбирая

$$
\delta=N^{-1 / 3} W^{-2 / 3} \log ^{1 / 2} W,
$$

получаем оченку

$$
\Sigma=\theta\left(N^{2 / 3} W^{1 / 3} \log ^{1 / 2} W\right)
$$

Поскольку для рассмотренных распределений математическое ожидание суммы площадей всех прямоугольников линейно зависит от $N W$ (или от $N$ в случае непрерывного распределения), полученные результаты свидетельствуют о хорошем качестве упаковок, получаемых с помощью алгоритма A1.

Алгоритм Al обладает тем недостатком, что для достижения оптимальной эффективности требуется заранее знать число прямоугольников (поскольку надо выбрать число $\delta$ в зависимости от $N$ ). В последнем разделе нашей статьи приведен алгоритм $\mathrm{A} 2$, который размещает прямоугольники по мере поступления и имеет те же асимптотические оценки ожидаемой незаполненной площади, а именно,

$$
\Sigma=O\left(N^{2 / 3} \log ^{1 / 2} N\right)
$$

для непрерывного распределения и

$$
\Sigma=O\left(N^{2 / 3} W^{1 / 3} \log ^{1 / 2} W\right)
$$

для дискретного распределения.

Интересно отметить, что если отказаться от требования, чтобы алгоритм работал в режиме on-line, то, как известно из [9], можно достичь лучших оценок для случая непрерывного равномерного распределения, а именно,

$$
\Sigma=O\left(N^{1 / 2} \log N\right) .
$$

Интересным открытым вопросом является достижимость подобной оценки в классе online алгоритмов.

\section{3. Доказательство теоремы 1}

Пусть зафиксированы число $N$ прямоугольников и высоты шельфов $\left\{r_{n}\right\}_{n=0}^{\infty}$. Каждый $i$-й прямоугольник определен своей высотой $h_{i}$ и шириной $w_{i}$, которые являются независимыми в совокупности случайными величинами. Для краткости будем обозначать через $\mathbf{h}$ набор $\left(h_{1}, \ldots, h_{N}\right)$, а через $\mathbf{w}$ набор $\left(w_{1}, \ldots, w_{N}\right)$. Пусть $N_{n}$ обозначает число прямоугольников, попавших в шельфы высоты $r_{n}$, то есть

$$
N_{n}=\left|\left\{h_{i} \mid r_{n+1}<h_{i} \leqslant r_{n}\right\}\right| \text {. }
$$

Заметим, что случайная величина $N_{n}$ не зависит от $\mathbf{w}$, а зависит только от $\mathbf{h}$.

Пусть $\left\{w_{i}^{n}, h_{i}^{n}\right\}_{i=1}^{N_{n}}-$ набор прямоугольников, упакованных в шельфы высоты $r_{n}$. 
Доказательство теоремы 1 . Так как $h_{i}$ имеет равномерное распределение, случайная величина $N_{n}$ имеет биномиальное распределение такое, что

$$
\mathbf{P}\left\{N_{n}=k\right\}=\left(\begin{array}{c}
N \\
k
\end{array}\right) p^{k}(1-p)^{N-k},
$$

где

$$
p=r_{n}-r_{n+1}, \quad 0 \leqslant k \leqslant N \text {. }
$$

Пусть $S_{n}$ - число шельфов высоты $r_{n}$, образовавшихся в процессе упаковки.

Используя для условного математического ожидания $\mathrm{E}(X \mid Y)$ случайных величин $X$ и $Y$ равенство

$$
\mathbf{E} X=\mathbf{E}(\mathbf{E}(X \mid Y))
$$

оценим ожидаемую пустую площадь:

$$
\begin{aligned}
\mathbf{E}\left(\sum_{n=0}^{\infty} r_{n} S_{n}-\sum_{i=1}^{N} w_{i} h_{i}\right) & =\mathbf{E} \sum_{n=0}^{\infty} r_{n} S_{n}-\frac{N}{4} \\
& =\mathbf{E} \sum_{n=0}^{\infty} r_{n} \mathrm{E}\left(S_{n} \mid \mathbf{h}\right)-\frac{N}{4}
\end{aligned}
$$

Для упаковки в шельфах заданной высоты применяется алгоритм BF, поэтому, используя известную для него оценку заполнения (см. [7]), получаем, что

$$
\mathbf{E}\left(\left(1 \cdot S_{n}-\sum_{i=1}^{N_{n}} w_{i}^{n}\right) \mid \mathbf{h}\right)=\theta\left(\sqrt{N_{n}} \log ^{3 / 4} N_{n}\right) \text {. }
$$

Отсюда, ввиду независимости $w_{i}^{n}$ от $\mathbf{h}$, получаем оценку для числа шельфов

$$
\begin{aligned}
\mathbf{E}\left(S_{n} \mid \mathbf{h}\right) & =\mathbf{E}\left(\sum_{i=1}^{N_{n}} w_{i}^{n} \mid \mathbf{h}\right)+\theta\left(\sqrt{N_{n}} \log ^{3 / 4} N_{n}\right) \\
& =\frac{N_{n}}{2}+\theta\left(\sqrt{N_{n}} \log ^{3 / 4} N_{n}\right) .
\end{aligned}
$$

Используя это соотношение, получаем, что математическое ожидание пустой площади равно

$$
\begin{aligned}
\Sigma & =\mathbf{E} \sum_{n=0}^{\infty} r_{n} \mathbf{E}\left(S_{n} \mid \mathbf{h}\right)-\frac{N}{4} \\
& =\mathbf{E} \sum_{n=0}^{\infty} r_{n}\left(\frac{N_{n}}{2}+\theta\left(\sqrt{N_{n}} \log ^{3 / 4} N_{n}\right)\right)-\frac{N}{4} \\
& =\sum_{n=0}^{\infty}\left(r_{n} \frac{N\left(r_{n}-r_{n+1}\right)}{2}+\mathbf{E} r_{n} \theta\left(\sqrt{N_{n}} \log ^{3 / 4} N_{n}\right)\right)-\frac{N}{4}
\end{aligned}
$$


В последнем равенстве мы использовали очевидное соотношение

$$
\mathbf{E} N_{n}=N\left(r_{n}-r_{n+1}\right) \text {. }
$$

Выбирая в качестве $\left\{r_{n}\right\}$ последовательность

$$
r_{n}=(1-\delta)^{n}, \quad 0<\delta<1,
$$

получаем, что

$$
\begin{aligned}
\Sigma & =\sum_{n=0}^{\infty}(1-\delta)^{n} \mathbf{E} \theta\left(\sqrt{N_{n}} \log ^{3 / 4} N_{n}\right)+\frac{N}{2(2-\delta)}-\frac{N}{4} \\
& \leqslant \log ^{3 / 4} N \sum_{n=0}^{\infty}(1-\delta)^{n} \theta\left(\mathbf{E} \sqrt{N_{n}}\right)+\frac{N}{2(2-\delta)}-\frac{N}{4}
\end{aligned}
$$

Для получения верхней оценки воспользуемся неравенством Йенсена

$$
\mathbf{E} \sqrt{N_{n}} \leqslant \sqrt{\mathbf{E} N_{n}}
$$

Обозначая

$$
p=r_{n}-r_{n+1}=(1-\delta)^{n} \delta
$$

получим неравенство

$$
\mathbf{E} \sqrt{N_{n}} \leqslant \sqrt{N p}
$$

Оценим в (1) сумму

$$
\begin{aligned}
\Sigma_{2} & =\sum_{n=0}^{\infty}(1-\delta)^{n} \theta\left(\mathbf{E} \sqrt{N_{n}}\right) \\
& \leqslant c \sum_{n=0}^{\infty}(1-\delta)^{n} \sqrt{N(1-\delta)^{n} \delta} \\
& =c \sqrt{N \delta}\left(1-(1-\delta)^{3 / 2}\right)^{-1},
\end{aligned}
$$

где $c$ - некоторая постоянная. Таким образом, получаем общую верхнюю оценку

$$
\Sigma \leqslant \frac{c \sqrt{N \delta} \log ^{3 / 4} N}{1-(1-\delta)^{3 / 2}}+\frac{N}{2(2-\delta)}-\frac{N}{4} .
$$

Далее получаем, что

$$
\begin{aligned}
\Sigma & \leqslant \frac{c \sqrt{N \delta} \log ^{3 / 4} N}{1-(1-\delta)^{3 / 2}}+\frac{N}{2(2-\delta)}-\frac{N}{4} \\
& =\sqrt{N} \log ^{3 / 4} N \frac{c \sqrt{\delta}}{1-(1-\delta)^{3 / 2}}+\frac{N}{2}\left(\frac{1}{2-\delta}-\frac{1}{2}\right) \\
& \leqslant \sqrt{N}\left(\frac{c \sqrt{\delta} \log ^{3 / 4} N}{1-(1-\delta)^{3 / 2}}+\frac{\delta \sqrt{N}}{4}\right) .
\end{aligned}
$$


Далее,

$$
\sqrt{N}\left(\frac{c \sqrt{\delta} \log ^{3 / 4} N}{1-(1-\delta)^{3 / 2}}+\frac{\delta \sqrt{N}}{4}\right) \leqslant \sqrt{N}\left(\frac{c \log ^{3 / 4} N}{\sqrt{\delta}}+\frac{\delta \sqrt{N}}{4}\right) .
$$

Таким образом, мы доказали, что

$$
\Sigma=O\left(\frac{\sqrt{N} \log ^{3 / 4} N}{\sqrt{\delta}}+\delta N\right) .
$$

Выбирая

$$
\delta=N^{-1 / 3} \log ^{1 / 2} N
$$

получаем оценку

$$
\Sigma=O\left(N^{2 / 3} \log ^{1 / 2} N\right)
$$

Получим теперь оценку снизу. Ясно, что

$$
\begin{aligned}
\Sigma_{2} \geqslant & \sum_{n=0}^{\infty}(1-\delta)^{n} \sum_{k:|k-N p| \leqslant 2 \ln N p \sqrt{N p}} \sqrt{k} \log ^{3 / 4} k\left(\begin{array}{l}
N \\
k
\end{array}\right) p^{k}(1-p)^{N-k} \\
\geqslant & \sum_{n=0}^{\infty}(1-\delta)^{n} \sqrt{N p-2 \ln N p \sqrt{N p}} \ln ^{3 / 4}(N p-2 \ln N p \sqrt{N p}) \\
& \times \sum_{k:|k-N p| \leqslant 2 \ln N p \sqrt{N p}}\left(\begin{array}{l}
N \\
k
\end{array}\right) p^{k}(1-p)^{N-k} .
\end{aligned}
$$

Используем известное неравенство для вероятностей больших уклонений [11]: для любого $0<a<1$

$$
\sum_{k:|k-N p|>N p a}\left(\begin{array}{l}
N \\
k
\end{array}\right) p^{k}(1-p)^{N-k} \leqslant 2 e^{-N p a^{2} / 3}
$$

Пусть $C_{0}-$ больший из двух корней уравнения

$$
\sqrt{C}=4 \ln C \text {. }
$$

Тогда, если $N p>C_{0}$, то

$$
\begin{aligned}
2 e^{-\ln ^{2} N p} & <\frac{1}{2} \\
N p-2 \ln N p \sqrt{N p} & >\frac{1}{2} N p .
\end{aligned}
$$

Выбрав

$$
a=\frac{2 \ln N p}{\sqrt{N p}}
$$


получим, что

$$
\begin{aligned}
\sum_{k:|k-N p| \leqslant 2 \ln N p \sqrt{N p}}\left(\begin{array}{l}
N \\
k
\end{array}\right) p^{k}(1-p)^{N-k} & =1-\sum_{k:|k-N p|>2 \ln N p \sqrt{N p}}\left(\begin{array}{l}
N \\
k
\end{array}\right) p^{k}(1-p)^{N-k} \\
& \geqslant 1-2 e^{-\ln ^{2} N p}
\end{aligned}
$$

Используя полученные неравенства и вспоминая, что

$$
p=\delta(1-\delta)^{n}
$$

получаем оценку

$$
\begin{aligned}
\Sigma_{2} & \geqslant \sum_{\left\{n: N p>C_{0}\right\}} \frac{(1-\delta)^{n} \sqrt{N p}}{2 \sqrt{2}} \log ^{3 / 4}(N p / 2) \\
& =\frac{\sqrt{N \delta}}{2 \sqrt{2}} \sum_{\left\{n: N p>C_{0}\right\}}(1-\delta)^{(3 / 2) n}\left(\ln \left(\frac{N \delta}{2}\right)-n \ln \frac{1}{1-\delta}\right)^{3 / 4} .
\end{aligned}
$$

Далее воспользуемся очевидным неравенством

$$
(1-\alpha)^{\beta} \geqslant 1-\alpha, \quad 0 \leqslant \alpha, \beta \leqslant 1,
$$

и получим, что

$$
\Sigma_{2} \geqslant \frac{\sqrt{N \delta}}{2 \sqrt{2}} \sum_{\left\{n: N p>C_{0}\right\}}(1-\delta)^{(3 / 2) n} \ln ^{3 / 4}\left(\frac{N \delta}{2}\right)\left(1+\frac{n \ln (1-\delta)}{\ln (N \delta / 2)}\right) .
$$

Условие $N p>C_{0}$ равносильно условию на $n$

$$
n \leqslant-\frac{\ln N \delta-\ln C_{0}}{\ln (1-\delta)}
$$

Обозначая

$$
K=\left[-\left(\ln N \delta-\ln C_{0}\right) / \ln (1-\delta)\right]
$$

и преобразуя сумму, получим, что

$$
\begin{aligned}
\Sigma_{2} \geqslant & \frac{\sqrt{N \delta}}{2 \sqrt{2}} \sum_{n=0}^{K}(1-\delta)^{(3 / 2) n} \ln ^{3 / 4}(N \delta / 2) \\
& \quad-\frac{\sqrt{N \delta}}{2 \sqrt{2}} \sum_{n=0}^{K}(1-\delta)^{(3 / 2) n} \ln ^{3 / 4}(N \delta / 2)\left(\frac{-n \ln (1-\delta)}{\ln (N \delta / 2)}\right) \\
\geqslant & \frac{\sqrt{N \delta}}{2 \sqrt{2}} \ln ^{3 / 4}\left(\frac{N \delta}{2}\right) \frac{1-(1-\delta)^{3 K / 2}}{1-(1-\delta)^{3 / 2}} \\
& \quad-\frac{\sqrt{N \delta}}{2 \sqrt{2}} \ln \frac{1}{1-\delta} \ln ^{-1 / 4}\left(\frac{N \delta}{2}\right) \sum_{n=0}^{K}(1-\delta)^{(3 / 2) n} n .
\end{aligned}
$$


Стандартными методами суммирования можно подсчитать, что

$$
\sum_{n=0}^{K} n(1-\delta)^{(3 / 2) n}=\frac{(1-\delta)^{3 / 2}\left(1-(1-\delta)^{3 K / 2}(K+1)+(1-\delta)^{3(K+1) / 2} K\right)}{\left(1-(1-\delta)^{3 / 2}\right)^{2}}
$$

С учетом этого находим, что

$$
\begin{aligned}
\Sigma_{2} \geqslant & \frac{\sqrt{N \delta}}{2 \sqrt{2}} \ln ^{3 / 4}\left(\frac{N \delta}{2}\right) \frac{1-(1-\delta)^{3 K / 2}}{1-(1-\delta)^{3 / 2}}-\frac{\sqrt{N \delta}}{2 \sqrt{2}} \ln \frac{1}{1-\delta} \\
& \times \ln ^{-1 / 4}\left(\frac{N \delta}{2}\right) \frac{(1-\delta)^{3 / 2}\left(1-(1-\delta)^{3 K / 2}(K+1)+(1-\delta)^{3(K+1) / 2} K\right)}{\left(1-(1-\delta)^{3 / 2}\right)^{2}} \\
\geqslant & \frac{\sqrt{N \delta}}{2 \sqrt{2}} \ln ^{3 / 4}\left(\frac{N \delta}{2}\right) \frac{1-\left(\ln C_{0} / \ln N \delta\right)^{3 / 2}}{1-(1-\delta)^{3 / 2}} \\
& \quad-\frac{\sqrt{N \delta}}{2 \sqrt{2}} \ln \frac{1}{1-\delta} \ln ^{-1 / 4}\left(\frac{N \delta}{2}\right) \frac{(1-\delta)^{3 / 2}}{\left(1-(1-\delta)^{3 / 2}\right)^{2}} \\
\geqslant & \frac{\sqrt{N}}{4 \sqrt{2 \delta}} \ln ^{3 / 4}\left(\frac{N \delta}{2}\right)\left(1-\left(\frac{\ln C_{0}}{\ln N \delta}\right)^{3 / 2}\right)-\frac{\sqrt{N \delta}}{2 \sqrt{2}} \ln \frac{1}{1-\delta} \ln ^{-1 / 4}\left(\frac{N \delta}{2}\right) \frac{1}{\delta^{2}} .
\end{aligned}
$$

Поскольку из условия теоремы следует, что $N \delta \rightarrow \infty$, при $\delta \rightarrow 0$ справедливы соотношения

$$
\begin{aligned}
\Sigma_{2} & =\Omega\left(\frac{\sqrt{N}}{\sqrt{\delta}} \ln ^{3 / 4}\left(\frac{N \delta}{2}\right)-\frac{\sqrt{N}}{\sqrt{\delta}} \ln ^{-1 / 4}\left(\frac{N \delta}{2}\right)\right) \\
& =\Omega\left(\frac{\sqrt{N}}{\sqrt{\delta}} \ln ^{3 / 4}\left(\frac{N \delta}{2}\right)\right) .
\end{aligned}
$$

Для оставшейся части суммы справедливо неравенство (см. (1))

$$
\frac{N}{2}\left(\frac{1}{2-\delta}-\frac{1}{2}\right)=\frac{N}{4}\left(\frac{\delta}{2-\delta}\right) \geqslant \frac{N}{8} \delta .
$$

Таким образом, получаем общую нижнюю оценку

$$
\Sigma=\Omega\left(\frac{\log ^{3 / 4}(N \delta)}{\sqrt{\delta}}+\delta \sqrt{N}\right) \sqrt{N}
$$

которая при некотором условии по порядку соответствует полученной верхней оценке. Для этого достаточно, чтобы выполнялось соотношение $1 / \delta=o(N \delta)$, что эквивалентно условию $1 / \delta=o(\sqrt{N})$.

В частности, при

$$
\delta=N^{-1 / 3} \log ^{1 / 2} N
$$

получаем нижнюю оценку

$$
\Sigma=\Omega\left(N^{2 / 3} \log ^{1 / 2} N\right)
$$

Доказательство теоремы 1 завершено. 


\section{4. Доказательство теоремы 2}

В настоящем разделе мы приводим доказательство теоремы 2, которое во многом сходно с доказательством теоремы 1. Напомним, что $w_{i}, i=1, \ldots, N$, имеют равномерное распределение $U(\{1,2, \ldots, W\})$.

Доказательство теоремы 2. Пусть $S_{n}$ - число шельфов высоты $r_{n}$, образовавшихся в процессе упаковки. Поскольку для упаковки в шельфах заданной высоты используется алгоритм BF, используя его свойства, получаем следуюшую оценку числа этих шельфов (см. [7]):

$$
\mathbf{E}\left(S_{n} W-\sum_{i=1}^{N_{n}} w_{i}^{n} \mid \mathbf{h}\right)=\theta\left(\sqrt{N_{n}} \log ^{3 / 4} W\right)
$$

откуда следует, что

$$
W \mathbf{E}\left(S_{n} \mid \mathbf{h}\right)=\frac{N_{n} W}{2}+\theta\left(\sqrt{N_{n}} \log ^{3 / 4} W\right) .
$$

Оценим теперь ожидаемую пустую площадь:

$$
\begin{aligned}
\Sigma & =\mathbf{E}\left(W \sum_{n=0}^{\infty} r_{n} S_{n}-\sum_{i=1}^{N} w_{i} h_{i}\right) \\
& =\mathbf{E} W \sum_{n=0}^{\infty} r_{n} S_{n}-\frac{N W}{4} \\
& =\mathbf{E} \sum_{n=0}^{\infty} r_{n}\left(\frac{N_{n} W}{2}+\theta\left(\sqrt{N_{n}} \log ^{3 / 4} W\right)\right)-\frac{N W}{4} \\
& =\sum_{n=0}^{\infty}\left(r_{n} \frac{N\left(r_{n}-r_{n+1}\right) W}{2}+\mathbf{E} r_{n} \theta\left(\sqrt{N_{n}} \log ^{3 / 4} W\right)\right)-\frac{W N}{4}
\end{aligned}
$$

Полагая, как и ранее,

$$
r_{n}=(1-\delta)^{n}, \quad 0<\delta<1,
$$

получаем, что

$$
\begin{aligned}
\Sigma & =\sum_{n=0}^{\infty}(1-\delta)^{n} \mathbf{E} \theta\left(\sqrt{N_{n}} \log ^{3 / 4} W\right)+\frac{N W}{2(2-\delta)}-\frac{W N}{4} \\
& =\log ^{3 / 4} W \sum_{n=0}^{\infty}(1-\delta)^{n} \theta\left(\mathbf{E} \sqrt{N_{n}}\right)+\frac{N W}{2(2-\delta)}-\frac{W N}{4}
\end{aligned}
$$

Обозначая

$$
p=r_{n}-r_{n+1}=(1-\delta)^{n} \delta
$$

и пользуясь неравенством Йенсена, находим, что

$$
\mathbf{E} \sqrt{N_{n}} \leqslant \sqrt{N p} .
$$


Поэтому

$$
\begin{aligned}
\Sigma_{2} & =\sum_{n=0}^{\infty}(1-\delta)^{n} \theta\left(\mathbf{E} \sqrt{N_{n}}\right) \\
& \leqslant c \sum_{n=0}^{\infty}(1-\delta)^{n} \sqrt{N(1-\delta)^{n} \delta} \\
& =c \sqrt{N \delta}\left(1-(1-\delta)^{3 / 2}\right)^{-1},
\end{aligned}
$$

где $c$ - некоторая постоянная. Таким образом, получаем общую верхнюю оценку

$$
\Sigma \leqslant \frac{c \log ^{3 / 4} W \sqrt{N \delta}}{1-(1-\delta)^{3 / 2}}+\frac{N W}{2(2-\delta)}-\frac{W N}{4} .
$$

Далее получаем, что

$$
\begin{aligned}
\Sigma & \leqslant \frac{c \log ^{3 / 4} W \sqrt{N \delta}}{1-(1-\delta)^{3 / 2}}+\frac{N W}{2(2-\delta)}-\frac{W N}{4} \\
& =\sqrt{N} \log ^{3 / 4} W \frac{c \sqrt{\delta}}{1-(1-\delta)^{3 / 2}}+\frac{N W}{2}\left(\frac{1}{2-\delta}-\frac{1}{2}\right) \\
& \leqslant \sqrt{N}\left(\frac{c \sqrt{\delta} \log ^{3 / 4} W}{1-(1-\delta)^{3 / 2}}+\frac{\delta \sqrt{N} W}{4}\right)
\end{aligned}
$$

и далее,

$$
\sqrt{N}\left(\frac{c \sqrt{\delta} \log ^{3 / 4} W}{1-(1-\delta)^{3 / 2}}+\frac{\delta \sqrt{N} W}{4}\right) \leqslant \sqrt{N}\left(\frac{c \log ^{3 / 4} W}{\sqrt{\delta}}+\frac{\delta \sqrt{N} W}{4}\right) .
$$

Таким образом, мы доказали, что

$$
\Sigma=O\left(\frac{\sqrt{N} \log ^{3 / 4} W}{\sqrt{\delta}}+\delta W N\right)
$$

Выбирая

$$
\delta=\left(N W^{2}\right)^{-1 / 3} \log ^{1 / 2} W,
$$

получаем оценку

$$
\Sigma=O\left(N^{2 / 3} W^{1 / 3} \log ^{1 / 2} W\right) .
$$

Получим теперь оценку снизу. Ясно, что

$$
\begin{aligned}
\Sigma_{2} & \geqslant \sum_{n=0}^{\infty}(1-\delta)^{n} \sum_{k:|k-N p| \leqslant \ln N p \sqrt{N p}} \sqrt{k}\left(\begin{array}{l}
N \\
k
\end{array}\right) p^{k}(1-p)^{N-k} \\
& \geqslant \sum_{n=0}^{\infty}(1-\delta)^{n} \sqrt{N p-\ln N p \sqrt{N p}} \sum_{k:|k-N p| \leqslant 2 \ln N p \sqrt{N p}}\left(\begin{array}{l}
N \\
k
\end{array}\right) p^{k}(1-p)^{N-k} .
\end{aligned}
$$


Пусть $C_{0}-$ больший из двух корней уравнения

$$
\sqrt{C}=4 \ln C \text {. }
$$

Тогда, если $N p>C_{0}$, то

$$
\begin{aligned}
2 e^{-\ln ^{2} N p} & <\frac{1}{2}, \\
N p-2 \ln N p \sqrt{N p} & >\frac{1}{2} N p .
\end{aligned}
$$

Используя то же неравенство для вероятностей больших уклонений, которое мы использовали при доказательстве теоремы 1 , получаем, что

$$
\sum_{k:|k-N p| \leqslant 2 \ln N p \sqrt{N p}}\left(\begin{array}{l}
N \\
k
\end{array}\right) p^{k}(1-p)^{N-k} \geqslant 1-2 e^{-\ln ^{2} N p} .
$$

Используя полученные неравенства и те же соображения, что и в доказательстве теоремы 1 , находим, что

$$
\begin{aligned}
\Sigma_{2} & \geqslant \sum_{\left\{n: N p>C_{0}\right\}} \frac{(1-\delta)^{n} \sqrt{N p}}{2 \sqrt{2}} \\
& =\sum_{n=0}^{\infty} \frac{(1-\delta)^{n} \sqrt{N p}}{2 \sqrt{2}}-\sum_{\left\{n: N p \leqslant C_{0}\right\}} \frac{(1-\delta)^{n} \sqrt{N p}}{2 \sqrt{2}} \\
& \geqslant \frac{\sqrt{N \delta}}{2 \sqrt{2}\left(1-(1-\delta)^{3 / 2}\right)}\left(1-\left(\frac{\ln C_{0}}{\ln N \delta}\right)^{3 / 2}\right) .
\end{aligned}
$$

Поскольку из условия теоремы следует, что $N \delta \rightarrow \infty$, получаем, что

$$
\Sigma_{2}=\Omega(\sqrt{N / \delta})
$$

Для оставшейся части суммы справедливо неравенство

$$
\frac{N W}{2}\left(\frac{1}{2-\delta}-\frac{1}{2}\right)=\frac{N W}{4}\left(\frac{\delta}{2-\delta}\right) \geqslant \frac{N W}{8} \delta .
$$

Таким образом, получаем общую нижнюю оценку

$$
\Sigma=\Omega\left(\frac{\log ^{3 / 4} W}{\sqrt{\delta}}+\delta \sqrt{N} W\right) \sqrt{N}
$$

которая по порядку соответствует полученной верхней оценке. В частности, при

$$
\delta=\left(N W^{2}\right)^{-1 / 3} \log ^{1 / 2} W
$$

получаем нижнюю оценку

$$
\Sigma=\Omega\left(N^{2 / 3} W^{1 / 3} \log ^{1 / 2} W\right)
$$

Доказательство теоремы 2 завершено. 


\section{On-line алгоритм}

Приведенный выше Алгоритм А1 обладал тем недостатком, что для достижения оптимальной эффективности требуется заранее знать число прямоугольников (так как значение $\delta$ выбиралось в зависимости от $N$ ). Обычное предположение об on-line алгоритмах таково, что число поступающих объектов заранее неизвестно. Таким образом, предложенные ранее алгоритмы нельзя считать оn-line алгоритмами.

В данном разделе приведен on-line алгоритм A2, имеющий ту же асимптотическую оценку ожидаемой незаполненной площади, что и алгоритм A1. Опишем этот алгоритм.

Мы будем разбивать прямоугольники на группы. Каждую группу будем упаковывать с помошью алгоритма Al со своим значением $\delta$, причем упаковка каждой новой группы будет начинаться с верхней границы последнего созданного при упаковке предыдущей группы шельфа. Пусть общее число прямоугольников равно $N$. Представим его в виде

$$
N=m+\sum_{i=1}^{K} n^{i}
$$

где $1 \leqslant m \leqslant n^{K+1}$, а $n$ - некоторый заданный целочисленный параметр (например, можно положить $n=2$ ). Первая группа прямоугольников будет состоять из $n$ прямоугольников, вторая - из $n^{2}$ и так далее, последняя $(K+1)$-я группа - из $m$ прямоугольников. Будем упаковывать $k$-ю группу с

$$
\delta_{k}=\left(n^{k}\right)^{-1 / 3} W^{-2 / 3} \log ^{1 / 2} W
$$

в дискретном случае и

$$
\delta_{k}=\left(n^{k}\right)^{-1 / 3} \log ^{1 / 2} n^{k}
$$

в случае непрерывного распределения.

Теорема 3. Для математического ожидания незаполненной площади $S$ при упаковке алгоритмом А2 справедлива оченка

$$
E S=O\left(N^{2 / 3} W^{1 / 3} \log ^{1 / 2} W\right)
$$

в дискретном случае и

$$
\mathbf{E} S=O\left(N^{2 / 3} \log ^{1 / 2} N\right)
$$

в непрерывном случае.

Доказательство. Доказательство проведем для непрерывного случая. Так как группы прямоугольников упаковываются независимо,

$$
S=\sum_{i=1}^{K} S\left(n^{i}, \delta_{i}\right)+S\left(m, \delta_{K+1}\right),
$$

где $S(N, \delta)$ - незаполненная площадь при упаковке $N$ прямоугольников с помощью алгоритма $\mathrm{A} 1$ с параметром $\delta$. В соответствии с утверждением теоремы 1 для любых $N$ и $\delta>0$ справедлива оценка математического ожидания незаполненной площади

$$
\mathbf{E} S(N, \delta) \leqslant C_{0} \frac{\sqrt{N} \log ^{3 / 4} N}{\sqrt{\delta}}+C_{1} \delta N,
$$


где $C_{0}$ и $C_{1}$ - некоторые постоянные. Тогда для $\mathbf{E}$ справедлива оценка

$$
\begin{aligned}
\mathbf{E} S= & \mathbf{E}\left(\sum_{i=1}^{K} S\left(n^{i}, \delta_{i}\right)+S\left(m, \delta_{K+1}\right)\right) \\
\leqslant & \sum_{i=1}^{K} C_{2} n^{2 i / 3} \log ^{1 / 2} n^{2 i / 3}+C_{0} \frac{\sqrt{m} \log ^{3 / 4} m}{\sqrt{\delta_{K+1}}}+C_{1} \delta_{K+1} m \\
\leqslant & C_{2} n^{2 / 3} \frac{n^{2 K / 3}-1}{n^{2 / 3}-1} \log ^{1 / 2} n^{2 K / 3} \\
& +C_{0} \frac{\log ^{3 / 4} m \sqrt{m} n^{(K+1) / 6}}{\log ^{1 / 4} n^{K+1}} \\
& +C_{1} \frac{m \log ^{1 / 2} n^{K+1}}{n^{(K+1) / 3}} \\
\leqslant & C_{2} n^{2(K+1) / 3} \log ^{1 / 2} n^{2 K / 3} \\
& +C_{0} \sqrt{n^{K+1}} n^{(K+1) / 6} \log ^{1 / 2} n^{K+1} \\
& +C_{1} \frac{n^{K+1} \log ^{1 / 2} n^{K+1}}{n^{(K+1) / 3}} \\
= & O_{n}\left(N^{2 / 3} \log ^{1 / 2} N\right)
\end{aligned}
$$

что доказывает формулу (4). Формула (3) доказывается аналогично.

Замечание 1. Как уже отмечалось выше, если отказаться от требования, чтобы алгоритм работал в режиме on-line, то для случая непрерывного равномерного распределения ширины прямоугольников можно достичь оценки

$$
\Sigma=O\left(N^{1 / 2} \log N\right) .
$$

Открытым вопросом является достижимость подобной оценки в класce on-line алгоритмов. Используя результат работы [7] (теорему 3) и доказательство нижней оценки в теореме 1, можно показать, что в классе шельфовых алгоритмов, где на втором этапе используется произвольная on-line эвристика, невозможно получить верхнюю оценку, лучшую чем $O\left(N^{2 / 3}\right)$.

\section{Список литературы}

1. Baker B. S., Brown D. J., Katseff H. P., A $5 / 4$ algorithm for two-dimensional packing. J. Algorithms (1981) 2, 348-368.

2. Baker B. S., Coffman E. J., Rivest R. L., Orthogonal packings in two dimensions. SIAM J. Computing (1980) 9 846-855.

3. Baker B. S., Schwartz J. S., Shelf algorithms for two dimensional packing problems. SIAM J. Computing (1983) 12, 508-525.

4. Brucker P., Scheduling algorithms. Springer, Berlin, 1998.

5. Coffman E. J., Garey M. R., Johnson D. S., Tarjan R. E., Performance bounds for level-oriented two-dimensional packing algorithms. SIAM J. Computing (1980) 9, 808-826. 
6. Coffman E. G., Jr., Garey M. R., Johnson D. S., Approximation algorithms for Bin-Packing-An updated survey. In: Algorithm design for computer system design. Springer, Berlin, 1984, pp. 49106.

7. Coffman E. G., Jr., Courcoubetis C., Garey M. R., Johnson D. S., Shor P. W., Weber R. R., Yannakakis M., Perfect packing theorems and the average-case behavior of optimal and online bin packing. SIAM Review (2002) 44, 95-108.

8. Csirik J., Johnson D. S., Kenyon C., Orlin J. B., Shor P. W., Weber R. R., On the Sum-of-Squares algorithm for Bin Packing. In: Proc. Annual ACM Symp. Theory of Computing. 2000, pp. 208-217.

9. Karp R. M., Luby M., Marchetti-Spaccamela A., A probabilistic analysis of multidimensional bin packing problems. In: Proc. Annual ACM Symp. Theory of Computing. 1984, pp. 289-298.

10. Kenyon C., Remila E., A near optimal solution to a two-dimensional cutting stock problem. Math. Operat. Research (2000) 25 645-656.

11. Motwani R., Raghavan P., Randomized algorithms. Cambridge Univ. Press, Cambridge, 1995.

12. Sleator D. D., A 2.5-times optimal algorithm for bin packing in two dimensions. Inf. Processing Lett. (1980) 10, 37-40.

13. R. E. Korf, Optimal rectangle packing: initial results. In: Proc. Intern. Conf. on Automated Planning and Scheduling. 2003, pp. 287-295.

Статья поступила 26.01.2005. 